\title{
Divided attention between simultaneous auditory and visual signals
}

\author{
ANNE-MARIE BONNEL \\ Centre National de la Recherche Scientifique, Marseille, France \\ and \\ ERVIN R. HAFTER \\ University of California, Berkeley, California
}

\begin{abstract}
Past studies of simultaneous attention to pairs of visual stimuli have used the "dual-task" paradigm to show that identification of the direction of a change in luminance, whether incremental or decremental, is "capacity-limited," while simple detection of these changes is governed by "capacity-free" processes. On the basis of that finding, it has been suggested that the contrast between identification and detection reflects different processes in the sensory periphery, namely the responses of magno- and parvocellular receptors. The present study questions that assertion and investigates the contribution of central processing in resource limitation by applying the dual task to a situation in which one stimulus is auditory and one is visual. The results are much the same as before, with identification demonstrating the tradeoff in performance generally attributed to a limited capacity but detection showing no loss compared with single-task controls. This implies that limitations on resources operate at a central level of processing rather than in the auditory and visual peripheries.
\end{abstract}

In divided attention, performance limitations depend on a variety of features of both the stimulus and the response (see Duncan, 1980, and Hirst, 1986, for review). Recently, Bonnel, Stein, and Bertucci (1992) showed that limitations may also depend on the nature of the psychophysical paradigm. They used the dual-task methodology to present concurrent visual stimuli in two paradigms, "detection" and "identification." Subjects responded separately to simultaneous side-by-side visual channels. In detection, signals were increments in luminance $\mathrm{S}(+)$ that could be added to each channel independently. In identification, signals were either increments $\mathbf{S}(+)$ or decrements $\mathrm{S}(-)$ that were added to each stimulus independently. Thus, in detection, subjects monitored for the presence of signals whereas in identification they identified the signs of signals. Results indicated no effect of concurrence when the task was to detect, but clear deficits when the task was to identify.

Greater understanding of divided attention in the dual task is revealed by "attention-operating characteristics" (AOCs), which plot joint performance as a function of attentional instructions (e.g., Hoffman \& Nelson, 1981;

This work was supported in part by a research grant ( 5 R01 DC 00087) from the National Institute on Deafness and Other Communication Disorders. We wish to thank Thomas O.Wickens for supplying his corrected version of the Dorfman and Alf maximum likelihood procedure for ROC analysis as well as Neil A. Macmillan and other reviewers of an earlier manuscript who provided helpful comments and suggestions. Correspondence should be addressed to A.-M. Bonnel, Department of Psychology, 3210 Tolman Hall, University of California, Berkeley, CA 94720 (e-mail: ambonnel@garnet.berkeley.edu).
Kinchla, 1969, 1980; Navon \& Gopher, 1979; Sperling \& Melchner, 1978a, 1978b). Figure 1 is a schematic example of such a plot. Vertical and horizontal axes indicate hypothetical performance on each channel in units of $d_{A}^{\prime}$ and $d_{\mathrm{B}}^{\prime}$. Solid symbols represent performance in the single tasks (A or B alone), and open symbols show data from the dual task ( $A$ and $B$ ). The legends describe attentional instructions in which subjects were told to pay a fixed proportion $(p)$ of attention to $\mathrm{A}$ and the rest $(1-p)$ to $B$. By definition, the AOC is the locus of points for all values of $p$. The two idealized AOCs drawn to illustrate extreme models of resource allocation assume that the subject follows instructions about $p$ accurately. The intersection of the dashed lines at the so-called independence point shows the prediction of a model in which there is no cost of divided attention or no resource limitation regardless of $p$. Conversely, the curved solid line illustrates a model in which a fixed resource (or capacity) is divided between inputs according to $p$. Here, the total resource $N$ (Luce, 1977) is defined as the total number of independent samples available. According to signal detection theory (SDT; Green \& Swets, 1966), $d^{\prime}$ increases with the square root of the number of independent samples, so that the predicted effects of $p$ are

$$
\left[d_{p N}^{\prime}\right]^{2}+\left[d_{(1-p) N}^{\prime}\right]^{2}=\left[d_{N}^{\prime}\right]^{2}
$$

From this, the relations between performance in the dual and single tasks as shown by the solid curve are

$$
d_{d u a l, A}^{\prime}=\sqrt{p} d_{\text {single }, A}^{\prime}
$$

and

$$
d_{d u a l, B}^{\prime}=\sqrt{1-p} d_{\text {single }, B}^{\prime}
$$




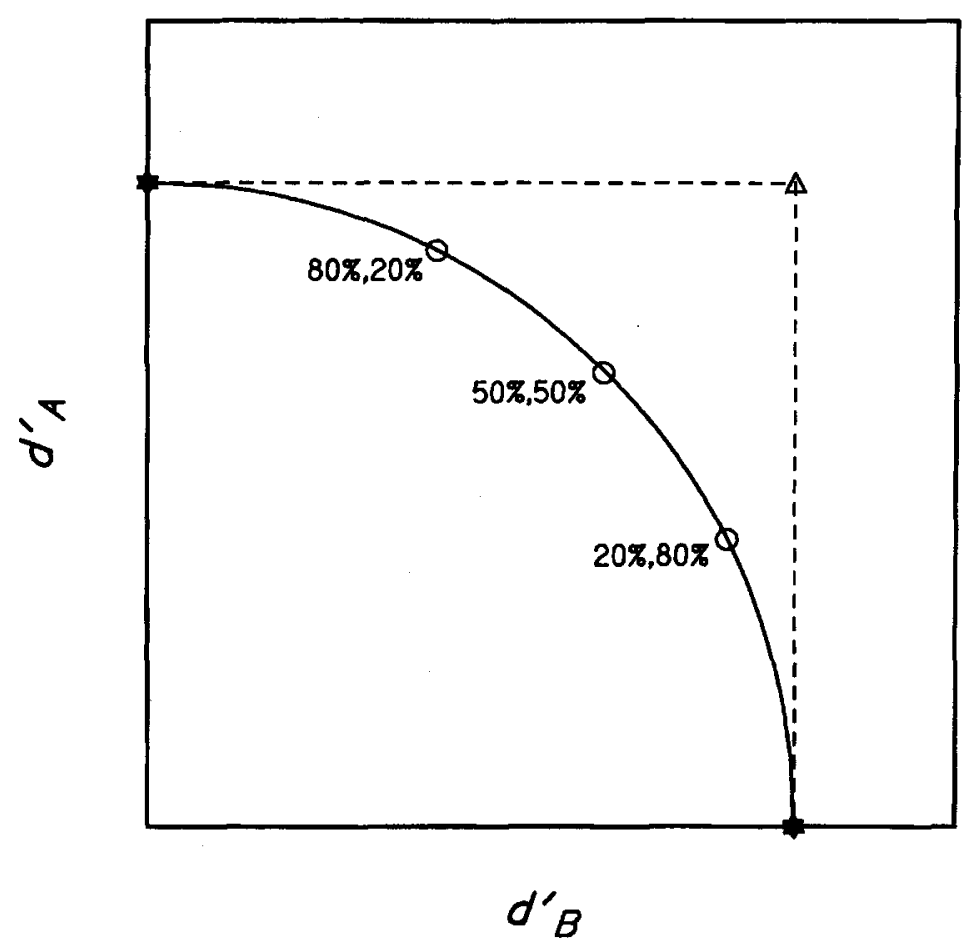

Figure 1. Schematic representation of a hypothetical attention operating characteristic (AOC) showing the joint performance (or sensitivity) in the dualtask paradigm. Solid symbols depict performance on either task alone. Open circles represent a case of resource limitation in which a fixed capacity is allocated to each task alone in the single task but divided according to instructions in the dual task. The open triangle represents a case of independence, where neither of the dual-task components affects the other.

In Bonnel et al. (1992), performance in detection fell close to the independence point, while that in identification was well fitted by the $\sqrt{ } N$-model.

The present experiment looked further into the results of Bonnel et al. (1992), with a particular focus on the question of where attention is limited. A longstanding issue is concerned with describing resources as a single, central, undifferentiated pool (Navon \& Gopher, 1979) versus describing them as multiple pools allotted to specific domains such as sensory modalities (Wickens, 1980). In order to address this issue, we chose to repeat the dualtask identification paradigm using two sensory channels, one auditory and one visual.

A second reason for using one auditory and one visual stimulus was to deal with a problem that can be raised concerning the logic of the dual task (see, e.g., Kahneman, 1973). It is assumed in a dual task that stimuli in the two channels are processed independently. Yet, Bonnel et al. (1992) found evidence of grouping in the identification task, with trial-by-trial accuracy being higher when $\mathrm{S}(+)$ was paired with $\mathbf{S}(+)$ or $\mathbf{S}(-)$ was paired with $\mathrm{S}(-)$.

A third advantage of using separate modalities is that it would eliminate a potential confound in the purely visual dual tasks in Bonnel et al. (1992). In an earlier study comparing detection and identification in an auditory sin- gle task, Macmillan (1971, 1973) suggested that the former relies on sensitivity to neural transients, whereas the latter is based on sustained activity. In line with this, Bonnel et al. raised the possibility that separate visual channels supplied the neural basis for the distinction between detection and identification found in the dual task. Two likely candidates for these pathways were the magnocellular visual pathways with special sensitivity to transients and the parvocellular pathways that carry a more sustained response to stimulus level. The possible confound derives from the fact that magnocellular receptors are more evenly distributed across the retina whereas parvocellular receptors are primarily foveal. Given that the visual stimuli of Bonnel et al. (1992) were separated by $14^{\circ}$ of arc, we wondered if the direction of gaze could have produced the tradeoff in performance indicated by the curved $A O C$ found in identification. Suppose that the instruction to pay $80 \%$ of attention to the left stimulus (A) and $20 \%$ to the right (B) led to a leftward bias in the direction of the subject's gaze, moving the high priority $(80 \%)$ stimulus onto the fovea and the low priority $(20 \%)$ stimulus more into the parafovea. Since both high- and low-priority channels would fall on magnocellular receptors, this would leave detection unaffected by instructions. However, in identification the high-priority stim- 
ulus would be better served by parvocellular receptors, thus accounting for differences between performance on high- and low-priority channels. This problem would not pertain to monitoring of separate modalities; with only a single visual stimulus to monitor, the focus of gaze would be irrelevant.

\section{EXPERIMENT 1}

\section{Method}

Stimulus sequence and environment. The temporal sequence for both single and dual tasks is shown in Figure 2. Each trial began with a 900-msec constant stimulus (pedestal). Signals, when present, appeared $440 \mathrm{msec}$ after the onset of the pedestals. At the end of the pedestals, subjects were given as much time as they wished to respond, after which there was a 1,500 -msec delay before beginning the next trial. Subjects worked in a sound-attenuating (80-dB) booth in constant low-level illumination.

Pedestals. Henceforth, we will refer to stimuli in the auditory and visual channels as $A$ and $V$, respectively. The pedestal (A) was a $500-\mathrm{Hz}$ tone presented binaurally through headphones. Its level, $\mathrm{S}(0) \mathrm{A}$, was $80 \mathrm{~dB}$ re $20 \mu \mathrm{Pa}$. The pedestal (V) was a 3-cm lighted circle made with the red phosphor at the center of a Samsung SyncMaster-3 noninterlaced monitor. From a viewing distance of approximately $60 \mathrm{~cm}$, this subtended a visual angle of approximately $2.9^{\circ}$. Its luminance, $\mathrm{S}(0) \mathrm{V}$, was $1.64 \mathrm{~mL}$ as measured with a Minolta CS-100 Chroma meter.

Signal levels. Signal levels were chosen on the basis of separate, single-channel identification tasks. On the basis of these calibra- tion experiments, $\mathbf{S}(+)$ and $\mathbf{S}(-)$ were set to be identical. Six subjects (paid undergraduate students) were trained until performance seemed constant. Stimulus levels on $\mathrm{A}$ and $\mathrm{V}$ were chosen to produce $d^{\prime}$ s of approximately 2.0 . Individual differences in sensitivities were such that auditory signals varied across the subjects from 2 to $7 \mathrm{~dB}$, while visual signals varied from 0.025 to $0.075 \mathrm{~mL}$.

Auditory signals were 20 -msec ramped ${ }^{1}$ increments, $\mathrm{S}(+)_{\mathrm{A}}$, or decrements, $S(-)_{A}$ added to the amplitude of the auditory pedestal. Visual signals were $16.6-\mathrm{msec}$ increments, $S(+)_{V}$, or decrements, $\mathrm{S}(-)_{\mathrm{V}}$, in illumination added to the visual pedestal. These were timed to coincide with the refresh rate of the video monitor.

The detection paradigm. A major difference between this study and that of Bonnel et al. (1992) was in the signals employed in the detection paradigm. Whereas they used only $\mathrm{S}(+)$, we opted for a mixture of $S(+)$ and $S(-)$ randomized within each block of trials. Not only would this help to equate the difficulty of stimuli in the two paradigms, but it would also provide a more vigorous test of the hypothesis that detection was based on change per se. Here, the distribution of signals was $25 \%, \mathrm{~S}(+) ; 25 \%, \mathrm{~S}(-) ; 50 \%, \mathrm{~S}(0)$. The three types of trials are illustrated in Figure 2. In the dual task, the stimuli for each channel were selected independently. Trials were evenly distributed over the nine possible stimulus combinations. Subjects were told to report any change in the pedestals. In order to be consistent with previous studies (Bonnel \& Miller, 1994; Bonnel, Possamai, \& Schmitt, 1987; Bonnel et al., 1992; Miller \& Bonnel, 1994), responses were made with two 6-point rating scales using a computerized mouse that moved cursors on vertical displays. The scale on the left was labeled "sound"; the one on the right, "light." The top and bottom categories on each scale represented highest subjective certainty, with labels marked from top to bottom as "3 CHANGE SURE," "2," "1," "1," "2," "3 NO CHANGE SURE." At the end of

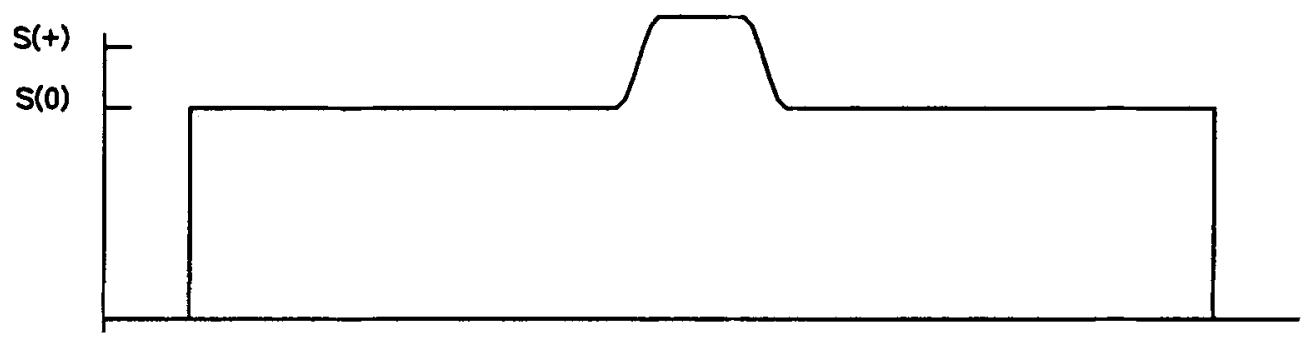

$S(0)$
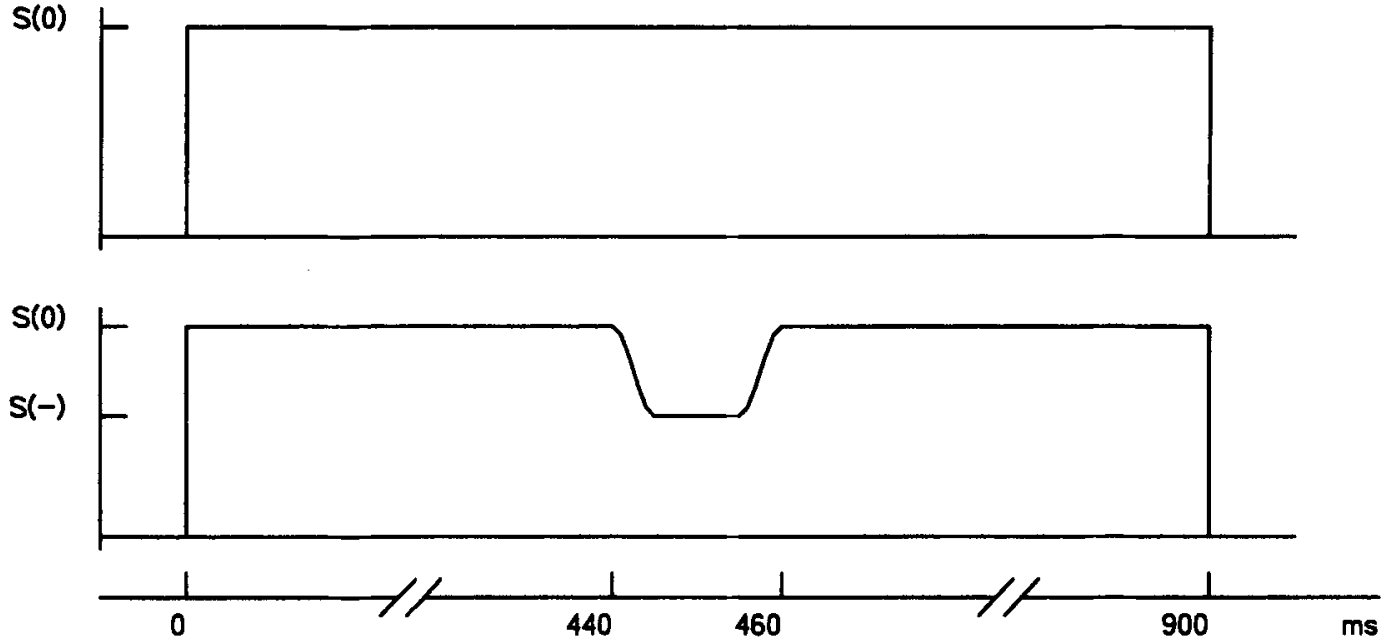

Figure 2. Temporal sequence for auditory and visual channels. Top and bottom panels illustrate signals used in identification and in detection. The middle panel illustrates a detection trial in which no signal is presented. 
a trial, feedback was given by highlighting the appropriate half of each scale.

The identification paradigm. In identification, $50 \%$ of the trials presented $\mathrm{S}(+)$ and $50 \%, \mathrm{~S}(-)$. Again, in the dual task, the stimuli for auditory and visual channels were selected independently. Subjects were told that there would be a change on every trial and that they were to identify the direction of this change. Again, the extremes of the scales represented highest subjective certainty. Now, the individual categories in each scale were labeled from top to bottom "3 HI SURE," "2," "1," "1," "2," "3 LO SURE." At the end of a trial, feedback was given by highlighting the appropriate half of each scale.

Manipulation of attention. In each single task, stimuli were presented only to the channel being tested. In the dual task, subjects were instructed to apportion their attention to the two channels according to the rule $p \%$ to the auditory channel and $(100-p) \%$ to the visual. For example, the instruction $80 \%, 20 \%$ meant "allocate $80 \%$ of attention to A and $20 \%$ to V." Other conditions included $20 \%, 80 \%$ and $50 \%, 50 \%{ }^{2}$ Each of these conditions was tested with 384 trials, identification in 8 blocks of 48 trials and detection in 12 blocks of 32 trials. Attentional instructions were constant throughout a block of trials and blocks were presented in random order. Subjects were free to choose which channel to respond to first; the order of responses was recorded for later analysis.

Data Analysis. The six-category ratings from each condition were used to construct a receiver operating characteristic (ROC). An iterative maximum-likelihood procedure from Dorfman and Alf (1969) was used to estimate ROC parameters as well as their variance. Sensitivity was evaluated using the index $d_{a}$, which is closely related to the area under the ROC $^{3}$ (see Macmillan \& Creelman, 1991, Equation 3.6). Parameters were estimated in two ways. First, in order to increase the stability of the estimates for the group data (Macmillan \& Kaplan, 1985), responses were pooled across subjects before plotting pooled ROCs. This procedure smooths irregularities caused by the tendencies of some subjects to not use the entire rating scale. Second, in order to allow for statistical testing, individual estimates were obtained from each subject's ROCs before averaging. These results are presented in Table 1. Agreement between these estimates was quite good, although the average estimates were consistently higher (see Swets \& Pickett, 1982).

\section{Results}

The resemblance between the AOCs from the bimodal dual task presented in Figure 3 and those with two visual stimuli (Bonnel et al., 1992) is striking. As before, although stimulus levels were the same for both paradigms, sensitivity as measured by the index $d_{a}$ was globally higher in detection than in identification. Statistical analysis confirmed a significant main effect of the paradigm $[F(5,1)=$ $15.03, p=.012$ ] based on a $2 \times 2 \times 4$ (channel $\times$ paradigm $\times$ attention) repeated measures analysis of variance (ANOVA) including all conditions of attention. In the same vein, joint detection scores fell along or even above the dotted line indicative of independence, whereas joint identification scores were lower than the single controls. These differences were tested by comparing performance in the two single tasks to the separate auditory and visual components of dual-task performance with the instruction $50 \%, 50 \%$. For detection, the apparent superiority of the divided-attention condition was not significant $[F(5,1)=.36, p=.575]$. However, identification performance dropped significantly in the $50 \%, 50 \%$ condition $[F(5,1)=10.47, p=.023]$, indicating a cost when the
Table 1

Signal Detection Analysis of Rating Data

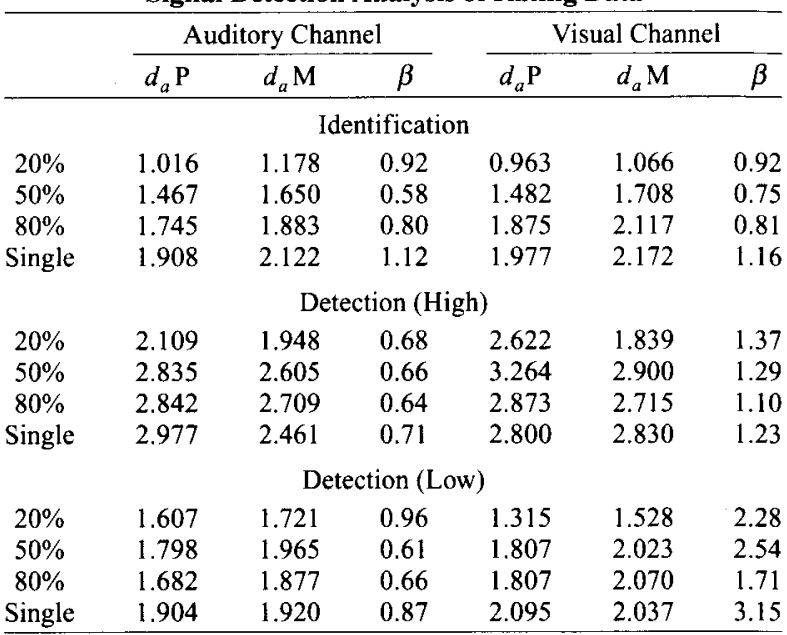

Note Estimate $d_{a} \mathrm{P}$ was obtained from pooled data and estimate $d_{a} \mathrm{M}$ was obtained by averaging the estimates obtained from individual subjects. Detection (high) presented same signal levels as in identification. Detection (low) presented lower levels set to produce the same singletask performance as identification.

subject had to identify the directions of changes in the two channels. These results are further confirmed by a significant interaction between paradigm (detection vs. identification) and attention (single vs. $50 \%)[F(5,1)=6.97$, $p=.046]$.

Instructions and sensitivity. The fact that subjects perform better in detection than in identification has led researchers (e.g., Bonnel et al., 1992; Klein, 1985; Macmillan, 1971, 1973) to speculate that the relevant stimuli in the two paradigms are fundamentally different. They have suggested that identification is based on sensitivity to sustained stimulus levels whereas detection reflects sensitivity to change per se. One should note that similar results accrue when both pedestal and signals are wideband noise (Macmillan, 1973), demonstrating that sensitivity to change, as used here, is more than just sensitivity to the spectral splatter. Figure 4 uses the logic of traditional SDT to explain why it is necessary to postulate the use of different kinds of information in the two paradigms. It compares performance for an "ideal observer" given the assumption that the relevant information is the stimulus level during the 20 -msec observation interval. Two assumptions from SDT are that (1) the internal representations of each level vary due to the addition of internal noise (Gaussian distributed with a mean $=0$ and a standard deviation $=\sigma$ ) and (2) the ideal observer applies a decision rule that is based on likelihood ratios. The three panels on the left show probability density distributions for the internal representations in each of three conditions. Panel A describes the condition used by Macmillan (1971) and Bonnel et al. for detection, with comparisons being between $S(0)$ and $S(+)$; panel $\mathrm{B}$ describes the condition used here for detection, 


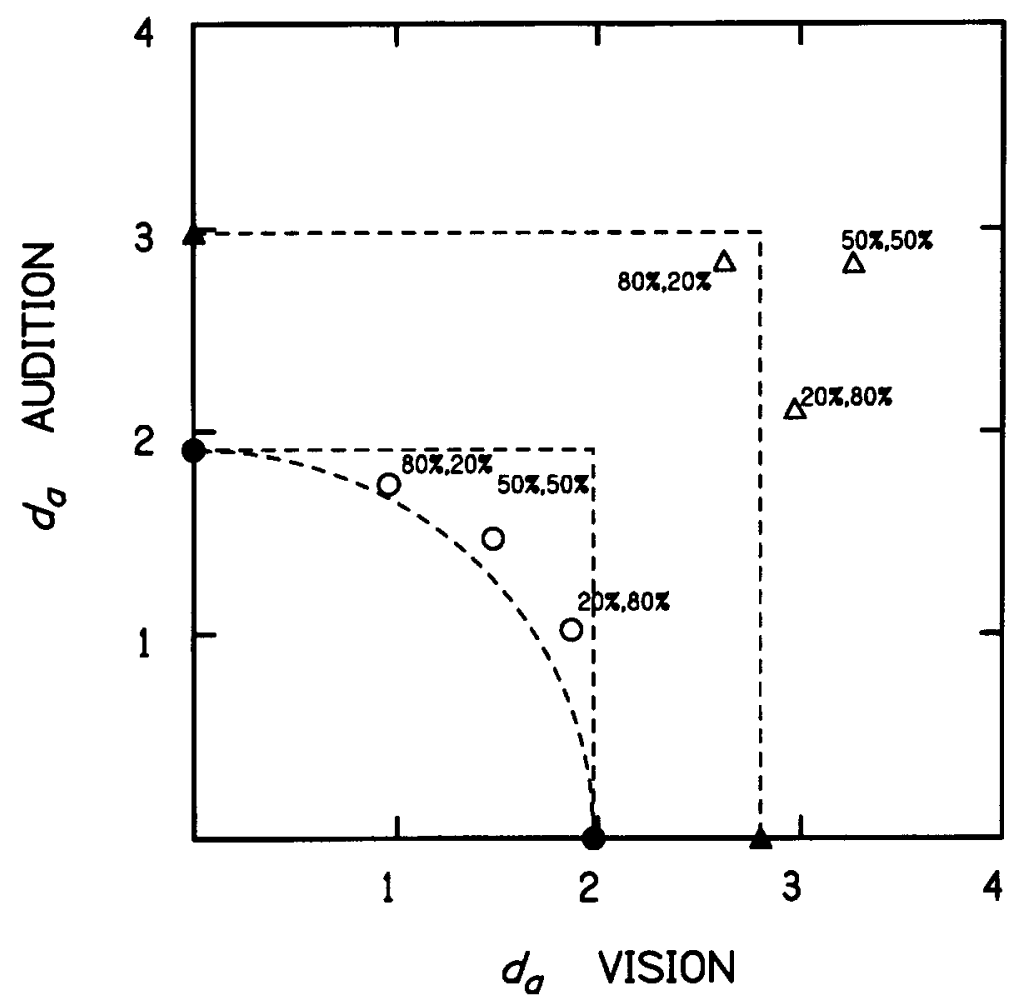

Figure 3. Experiment 1. Comparison of detection and identification performances using identical signal levels (pooled data). Attention operating characteristics (AOCs) show sensitivity $d_{a}$ for each channel alone and in conjunction. Solid points represent performance on the single channels alone. Open points are from the dual task, with the individual labels describing how subjects were instructed to share attention between channels: percent auditory, percent visual. Triangles are for detection, circles are for identification. The dashed curve represents the AOC predicted for identification from Equation 2.

with comparisons being between $S(0)$ and $S(+)$ or $S(-)$; panel $\mathrm{C}$ describes the condition used here for identification, with comparisons being between $S(-)$ and $S(+)$. For this example, the stimulus levels (in units of $z$ ) are $-1,0$, and +1 for $S(-), S(0)$ and $S(+)$, respectively. Note that for panels $A$ and $C$, likelihood ratios increase monotonically from left to right, whereas in B they are greatest at the extremes and decrease toward a minimum at the center. The large panel on the right shows performance calculated as the areas under the hypothetical ROCs for the three conditions. They are shown for absolute signal levels ranging from $|0|$ to $|2.5|$. We see that an SDT model based on stimulus level calls for higher performance in identification (Condition $\mathrm{C}$ ) than in detection with signals that are only increments (Condition A), an effect that is even greater when the signal in detection can be either an $\mathrm{S}(-)$ or $\mathrm{S}(+)$ (Condition $\mathrm{B}$ ). Yet, our results were just the opposite. It was the finding that this prediction was not borne out that led to postulation of hypersensitivity to transients.

All-or-none switching. The curved AOC is indicative of a division of resources between the two channels. However, one can imagine a strategy in which the subject in- terprets the instructions by switching between trials, allocating all resources to one channel or to the other. If such all-or-none switching were to occur, accuracy on one task would be associated with inaccuracy on the other. This strategy predicts a negative correlation in trial-bytrial analysis of the joint probability of correct responses in the two channels (Sperling \& Melchner, 1978a, 1978b). To test for all-or-none switching, each trial was tabulated in a $2 \times 2$ table; rows indicated the accuracy of the auditory task and columns indicated the accuracy on the visual task. Separate tables were constructed for each attention condition $(20 \%, 80 \% ; 50 \%, 50 \%$; and $80 \%, 20 \%)$. Correlations between accuracies on the two channels are shown in Table 2 as $\varphi$ coefficients (Hays, 1969, p. 604) that range from -1 (total negative dependence) to +1 (total positive dependence). Each $\varphi$ was based on 384 observations pooled across 6 subjects and none was significant. ${ }^{4}$ By showing that a correct response to one channel does not predict an error on the other, this analysis rejects an all-or-none switching model.

Perceptual grouping. As described in the introduction, the validity of the dual task rests on the assumption that the two channels are processed independently. Although 

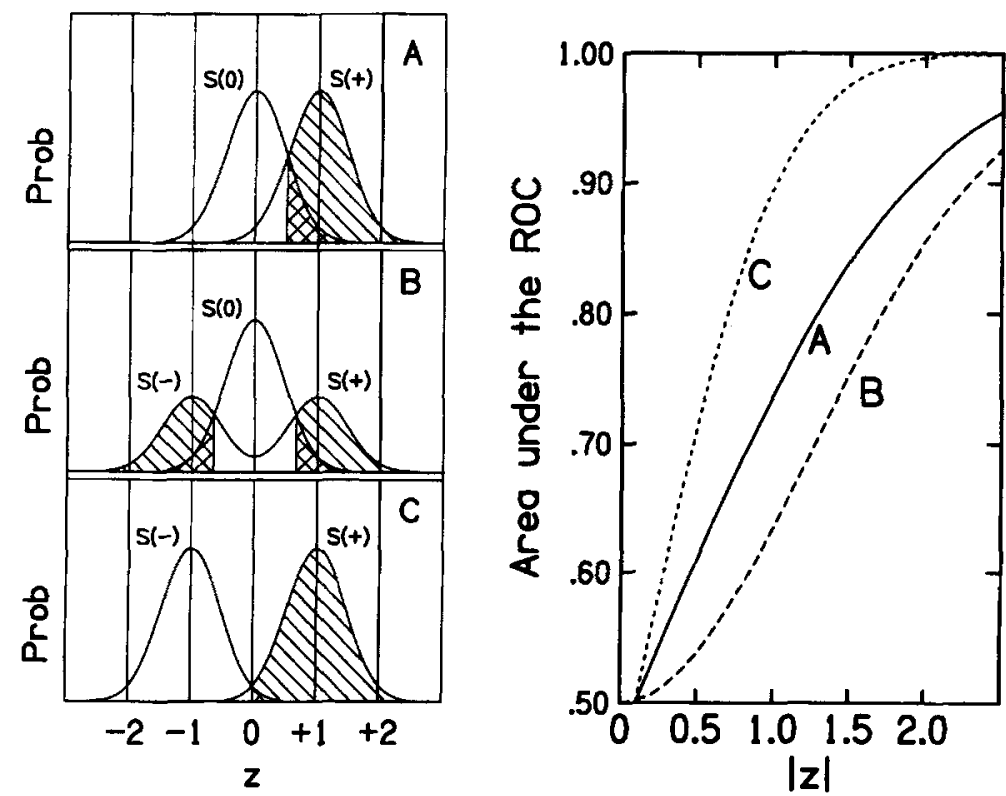

Figure 4. Predictions from a traditional SDT argument for the conditions discussed here. Drawings in the leftmost panels represent probability density distributions for signal + noise and noise alone. Panels $A$ and $B$ describe conditions used to measure detection while panel $C$ describes identification. Curves on the right panel are areas under the ROCs for each of these conditions plotted as a function of signal level.

the use of separate sensory modalities makes such grouping unlikely, this aspect can be examined by comparing the joint probabilities of being correct on both channels when the two signals are the same or different. To this end, all of the identification data were split into separate matrices according to the relation between the two channels $(+,+),(-,-),(+,-),(-,+)$. The joint percentage of correct responses was computed for each matrix. Unlike the previous visual/visual dual task (Bonnel et al., 1992), congruent signals were not identified more accurately, as can be seen from Table 3 . This table also shows that in detection, the probability of being correct on both channels was not different when one or two changes were presented. In demonstrating that processing of the auditory and visual channels was truly independent, this result supports the contention that a dualtask analysis is in order.

Effects of attention on response criteria. It has often been argued that measures of the effects of attention on detectability may be biased by shifts in the response criterion (e.g., Müller \& Findlay, 1987). For the present data, response biases as defined in SDT were examined by collapsing the six response categories into simple $2 \times$ 2 matrices. In the detection task, data in the higher three categories were summed and called "change," and those in the lower three categories were summed and called "no change." Similarly, the data from the identification task were collapsed into categories called "increment" and "decrement," respectively. Indices of bias, $\beta$, are presented in Table 1 as geometric means of the individual values calculated from each subject. The comparison of interest indicates that there was no systematic relation between $\beta$ and attention instructions.

\section{EXPERIMENT 2}

The decision to use identical stimulus levels for detection and identification in Experiment 1 led to unusually

Table 2

Observed $\varphi$ Coefficients as a Function of Attention and Psychophysical Paradigm

\begin{tabular}{|c|c|c|c|c|c|c|}
\hline & \multirow{2}{*}{\multicolumn{2}{|c|}{ Experiment 1}} & \multirow{3}{*}{$\frac{\text { Experiment } 2}{\text { Detection (Low) }}$} & \multicolumn{3}{|c|}{ Experiment 3} \\
\hline & & & & \multicolumn{2}{|c|}{ Identification } & Detection \\
\hline & Identification & Detection (High) & & Free Response Order & Constrained Response Order & High Low \\
\hline $20 \%, 80 \%$ & .014 & .075 & .065 & -.015 & .098 & $.184^{*}-.003$ \\
\hline $50 \%, 50 \%$ & .062 & .031 & .023 & .007 & $.058 / .032 \ddagger$ & $.268 \dagger \quad .007$ \\
\hline $80 \%, 20 \%$ & .021 & .050 & -.007 & .136 & .080 & $.189^{*}-.065$ \\
\hline
\end{tabular}

Note $-{ }^{*} p<.01 . \quad{ }^{\dagger} p<.001 . \quad{ }^{\ddagger}$ Respond first to auditory channel/first to visual channel. Detection (high) presented same signal levels as in identification. Detection (low) presented levels chosen to produce the same single-task performance as in identification. 


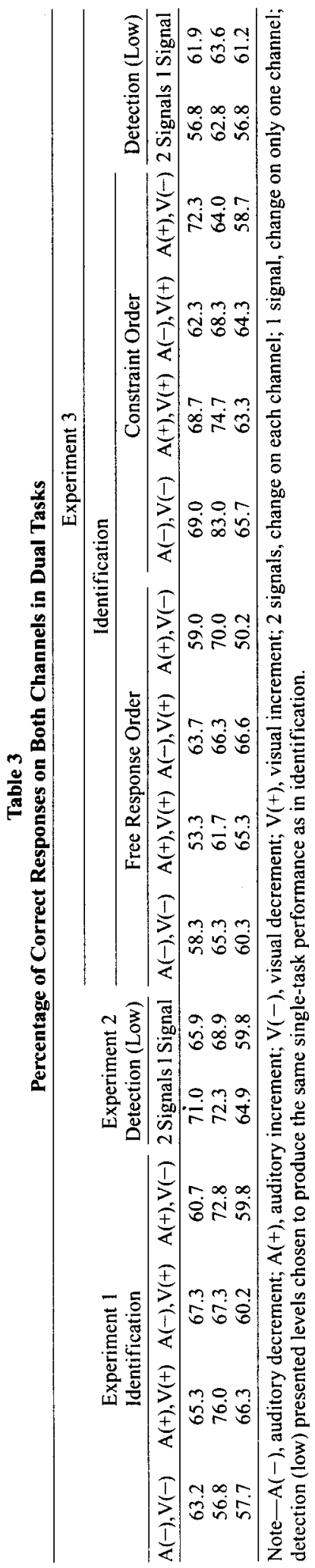

high $d^{\prime}$ values in detection. Generally, this is not satisfactory because small perturbations in the false alarm rate due to nonsensory factors can have an inordinate influence on the estimate of performance. Also, with very high levels, the subjects rarely use the entire rating scale, making an analysis based on the ROC curve problematic. The high levels also raise a potential problem for the discussion of shared attention since the so-called degenerate $\mathrm{AOC}$ exemplified by the dashed lines might reflect a kind of ceiling effect in which resources are not needed for extremely easy judgments. For all of these reasons, it was decided to retest detection with signal levels chosen to produce performance in the single task more like that found in identification.

\section{Method}

This experiment was identical to Experiment 1 with the exception of signal levels. Here, the visual signals used for the 6 subjects from the previous experiment ranged from $0.01 \mathrm{~mL}$ to $0.035 \mathrm{~mL}$ for both $\mathrm{S}(+)$ and $\mathrm{S}(-)$; auditory signals ranged from 2 to $3 \mathrm{~dB}$ for both $S(+)$ and $S(-)$. Henceforth, we will refer to this paradigm as detection (low).

\section{Results}

Figure 5 shows the AOC for detection with lower signal levels using the $d_{a}$ index of sensitivity described earlier. The results again failed to show an effect of capacity limitation. A $2 \times 4$ (channel, attention) ANOVA showed no significant effect of attention $[F(5,1)=0.34, p=$ .587]. Table 2 shows $\varphi$ coefficients between accuracies on the two channels for the grouped data. Again, the lack of negative correlation ${ }^{5}$ demonstrates that resources were not switched between trials. Table 3 shows that as with Experiment 1, there was no evidence of response grouping. The probability of being correct on both channels was slightly increased when two changes were presented, but this tendency was not significant. Finally, estimated response bias, shown in Table 1, showed no systematic effects on $\beta$.

One may conclude from these data that ceiling effects were not a factor in the original results. This reinforces the conclusion from Experiment 1 that resources were limited for identification but not for detection.

\section{EXPERIMENT 3}

An implicit assumption in Experiments 1 and 2 is that the selective cost of attention in identification but not in detection stems from differences between the sensory processing of transients and sustained levels. However, one can imagine potential artifacts that might also have affected both paradigms differentially. For one, "response interference" associated with the use of two simultaneous 6-point rating scales (with 10 response criteria) might have raised the overall cognitive load in a way that had a differential effect on the more demanding task. Second, there was the possibility that differences might have stemmed 


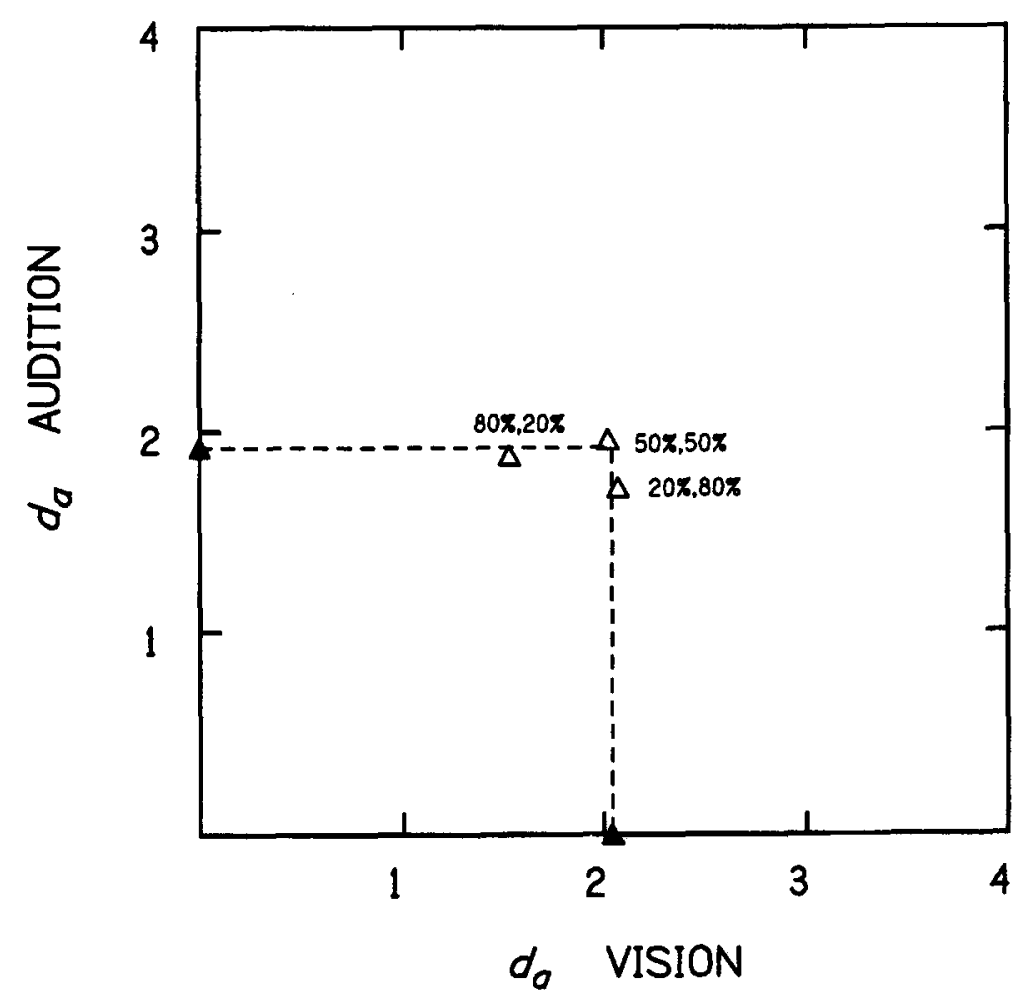

Figure 5. Experiment 2. Attention operating characteristic (AOC), observed in detection with lower signal levels chosen to produce the same single-task performance as in identification. In other respects similar to Figure 3.

from the labels on the response scales. Although the stimuli were explained to subjects in detail through the use of drawings that illustrated pedestals and signals (Figure 2), we worried that the labels on the response scales, which were "CHANGE" and "NO CHANGE" in detection and "HI" and "LO" in identification, might have induced them to make relative judgments in the first but absolute judgments in the second. Third, we observed that subjects left to respond freely nearly always responded first to the highpriority channel. Thus, performance on the low-priority channel in the more demanding task might have been reduced due to the use of an older and potentially weaker "trace memory" (Durlach \& Braida, 1969; Egeth, 1967).

Experiment 3 was designed to examine the importance of these potential influences. Experiments 1 and 2 would be repeated with a simpler response, using a twobutton box instead of the more complex 6-point rating scale; in addition, the labels used in identification would be changed to "UP" and "DOWN" to induce a more relative strategy. In addition, the effects of response order on identification would be tested by use of instructions that forced the subject to respond first to the low-priority channel.

\section{Method}

For this experiment, the computer/mouse rating scales were replaced with a box with two buttons for the auditory task and two for the visual. For detection, these were labeled "CHANGE" and "NO CHANGE." For identification the labels were "UP" and "DOWN." The
3 subjects had previously served in Experiments 1 and 2. In a first step, all of the earlier conditions were replicated with the simpler response with a free order of responding. After finishing the replication described above, the importance of response order in the identification paradigm was tested in additional sessions. In the unbalanced conditions $(80 \%, 20 \%$ and $20 \%, 80 \%)$, subjects were required to always respond first to the low-priority ( $20 \%$ channel). In the $50 \%, 50 \%$ condition, the channel (auditory or visual) to receive the first response alternated from block to block.

Data Analysis. As discussed above, the three-distribution modei of detection illustrated in Figure 4 is fundamentally wrong because it cannot account for the fact that performance is actually better in detection than in identification. Rather, we would argue that the appropriate analysis for computing $d$ 's for detection should be based on the assumption that change per se is unsigned. As such, the essential identity of the $S(-)$ and $S(+)$ signals argues for an SDT model based on a single signal + noise distribution. This allows one to use the more traditional model of $d^{\prime}$ found in Elliott's (1964/1988) tables. As before, pooled and average data, presented in Table 4, were in close agreement.

\section{Results}

Response interference. Figure 6 shows results from the replication of the first experiments with two button responses. In the left panel, stimulus levels were identical in detection and in identification; in the right panel, the levels were reduced to produce detection performance that matched the identification performance. Square AOCs are constrained to pass through the appropriate single-task points. The curved AOC passing through the single-task points for identification represents the locus of points 
Table 4

Signal Detection Analysis of Yes/No Data

\begin{tabular}{|c|c|c|c|c|c|c|}
\hline & \multicolumn{3}{|c|}{ Auditory } & \multicolumn{3}{|c|}{ Visual } \\
\hline & $d^{\prime} \mathrm{P}$ & $d^{\prime} \mathrm{M}$ & $\beta$ & $d^{\prime} \mathrm{P}$ & $d^{\prime} \mathrm{M}$ & $\beta$ \\
\hline \multicolumn{7}{|c|}{ Identification (Free Response Order) } \\
\hline $20 \%$ & 1.32 & 1.41 & 1.59 & 1.38 & 1.47 & 0.85 \\
\hline $50 \%$ & 2.26 & 2.31 & 0.89 & 1.98 & 2.16 & 1.32 \\
\hline $80 \%$ & 2.64 & 2.70 & 1.46 & 2.44 & 2.58 & 0.64 \\
\hline Single & 3.11 & 3.24 & 1.45 & 2.80 & 2.87 & 0.93 \\
\hline \multicolumn{7}{|c|}{ Identification (Constrained Response Order) } \\
\hline $20 \%$ & 1.28 & 1.58 & 1.79 & 1.08 & 1.36 & 0.85 \\
\hline $50 \%$ & 1.86 & 2.08 & $1.20 / 1.75+$ & 2.36 & 2.48 & $1.07 / 1.07+$ \\
\hline $80 \%$ & 2.72 & 2.92 & 0.65 & 2.72 & 2.79 & 1.94 \\
\hline Single & 3.11 & 3.24 & 1.45 & 2.80 & 2.87 & 0.93 \\
\hline \multicolumn{7}{|c|}{ Detection (High) } \\
\hline $20 \%$ & 2.77 & 2.99 & 0.49 & 2.90 & 3.24 & 1.76 \\
\hline $50 \%$ & 3.39 & 3.59 & 0.63 & 3.93 & 4.17 & 1.00 \\
\hline $80 \%$ & 3.93 & 3.99 & 0.84 & 4.20 & 4.24 & 1.57 \\
\hline Single & 3.63 & 3.85 & 0.64 & 4.38 & 4.46 & 1.49 \\
\hline \multicolumn{7}{|c|}{ Detection (Low) } \\
\hline $20 \%$ & 1.43 & 1.58 & 1.29 & 1.11 & 1.48 & 2.05 \\
\hline $50 \%$ & 2.47 & 2.60 & 1.16 & 2.30 & 2.44 & 3.17 \\
\hline $80 \%$ & 2.68 & 2.81 & 1.34 & 2.44 & 2.47 & 4.87 \\
\hline Single & 2.42 & 2.71 & 0.58 & 2.49 & 2.61 & 4.29 \\
\hline
\end{tabular}

Note--Estimate $d^{\prime} \mathrm{P}$ was obtained from pooled data and estimate $d^{\prime} \mathrm{M}$ was obtained by averaging the estimates obtained from individual subjects. Detection (high) presented same signal levels as in identification. Detection (low) presented lower levels set to produce the same singletask performance as in identification.

computed as a function of $p$ from Equation 2. This pattern of data captures the essence of the previous results, although it is not clear how one should interpret points that fall along the dotted lines, where the instructions to pay unequal attention to the two channels indicate no loss on the $80 \%$ channel but a lowering of performance on the $20 \%$ channel. This problem has been seen before (Gopher \& Navon, 1980; Navon, Gopher, Chillag, \& Spitz, 1984; Regan, 1982; Schneider \& Fisk, 1982). Perhaps points along the arms of the square AOC represent a strategy in which the subject interprets the unbalanced instruction to mean that performance "should be" less on the low-attention channel and responds to it inefficiently, using less than the available capacity (Navon, 1985). Whatever the case, such effects are difficult to reconcile with any model based on constant capacity.

Statistical analysis further indicated that concerns over response interference were unfounded. Indeed, comparisons between these data and those from the same 3 subjects in Experiments 1 and 2 revealed no significant differences between them. In a $2 \times 3 \times 2 \times 4$ (channel, paradigm, response, attention) repeated measures ANOVA, there was no significant effect of the response factor (ratings vs. yes/no) $[F(2,1)=3.60, p=.198]$. Thus, possible reduction of the cognitive load by adopting two-button boxes had no effect on the primary results; neither did a change of label for the response boxes. A full-scale analysis across all of the data from all three experiments clearly showed a significant interaction between para- digm (detection vs. identification) and attention (single vs. divided conditions) $[F(4,2)=8.93, p=.033]$, which is indicative of different mechanisms.

Trace memory. Figure 6 also describes the effect of constraining the order of response in identification. The data points are plotted as + 's. We see that responding first to the low-priority channels in the unbalanced conditions and to an arbitrarily assigned channel in $50 \%, 50 \%$ had no effect on accuracy or on the allocation of resources. When applied to the overall identification data from this experiment, a $2 \times 2 \times 4$ (channel, response, attention) repeated measures ANOVA confirmed that there was no significant effect of the response (free or constrained order) $[F(2,1)=.11, p=.774]$. Thus, as with response interference, we can discard trace memory as a determinant of the tradeoff found in identification.

Actual allocation of resources. The shapes of the AOCs we observed in identification clearly showed that resources were shared in a manner monotonically related to attentional instructions. However, one wonders how well subjects were able to interpret what was meant, for example, by " $80 \%$ of attention." The AOC is not the most sensitive representation for answering this question. Instead, Figure 7 plots $\left[d^{\prime}\right]^{2}$ as a function of the instructed values of $p$ for each channel in the form of performance resource functions (PRFs) ${ }^{6}$ The data are from Experiments 1 and 3. The model described in Equation 2 predicts that these data should lie on the straight line connecting $0 \%$ to $100 \%$ (single tasks) to the extent that subjects accurately followed the instructions. Although we see that overall performance was slightly lower than predicted for the unbalanced conditions, these functions fall close to the predictions of the sample-size model described by Equation 2.

Response biases, contingencies, and perceptual grouping. As with Experiments 1 and 2, the data in Experiment 3 were subjected to analysis of contingencies ${ }^{7}$ (see Table 2), perceptual grouping (see Table 3), and response bias (see Table 4). As before, there was no significant evidence of negative contingencies, response biases, or perceptual grouping.

\section{GENERAL DISCUSSION}

The primary result of this study is that the pattern of results found in the purely visual dual task of Bonnel et al. (1992) was repeated when the task was bisensorythat is, when one channel was auditory and one was visual. A major goal of the study was to evaluate potential confounds in the earlier work. One was that subjects might have directed their gaze more toward the highpriority location, placing the low-priority location more in the parafovea. Since there is no such competition for physical orientation between auditory and visual channels, the current data add further support to the argument that the tradeoff in sensitivity observed in identification was due to resource allocation. A second potential confound arose from their use of $\mathrm{S}(+)$ only as the signal in detec- 


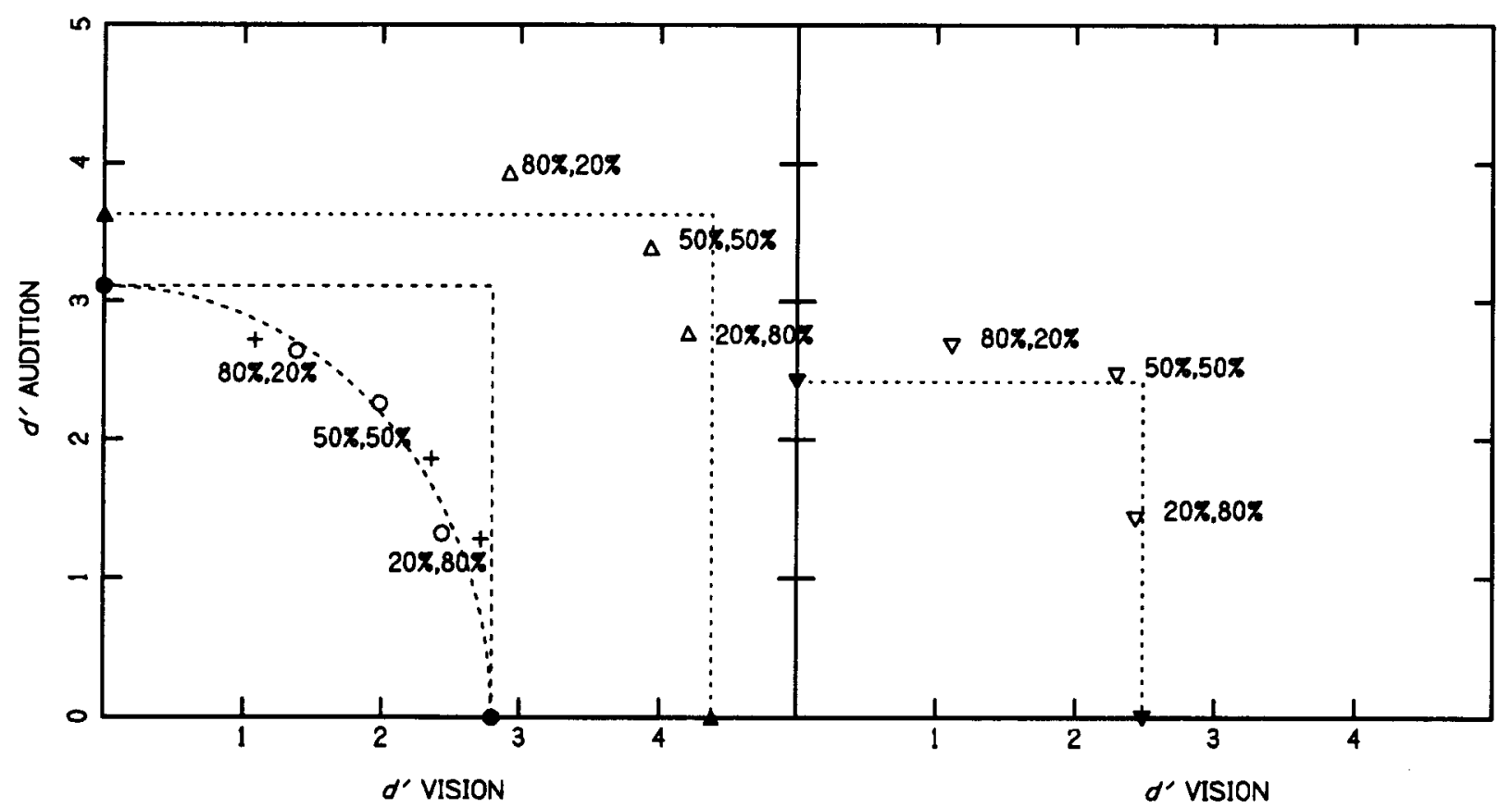

Figure 6. Experiment 3. Attention operating characteristics (AOCs), observed in the replication of Experiments 1 and 2 with a twobutton response. Left panel: Up-pointing triangles represent detection with high signal levels; circles represent identification with freeorder of responding; crosses represent identification with a constrained order of responding. Right panel: Down-pointing triangles represent detection with low signal levels. In other respects similar to Figures 3 and 5.

tion, since this introduced a difference in stimulus complexity between the two paradigms. However, this is dispelled by the essential replication of the earlier results by using signals that were a random mixture of $S(+)$ and $\mathrm{S}(-)$. Detection of change, as used here, implies detection of unsigned transients whose internal representations carry no information about the direction of change. The effectiveness of the mixture of $\mathrm{S}(+)$ and $\mathrm{S}(-)$ supports this view since signed transients could also be used for identification, making it more efficient than detection, as described in Figure 4. A third potential problem with the data from Bonnel et al. (1992) was the presence of perceptual grouping in identification, which could invalidate the assumption of independence in the dual-task paradigm. Since there was no evidence of such grouping in the auditory/visual data, the present data lend strong support to the view that identification in the dual task requires divided resources.

The effects of other potential artifacts also proved to be negative. First, the fact that lower stimulus levels in detection did not introduce signs of limited capacity discounted fears of a ceiling effect. Similarly, there was no effect of reducing response complexity by switching from a rating scale to a simple two-button response. Finally, tests with constraints on the order of response showed that there was no impact on performance on the low-priority channel due to the normal tendency of subjects to respond first to the high-priority channel.

The present study clearly shows that the distinction between capacity-free detection and capacity-limited iden- tification in the dual task occurs when the channels represent two different sensory modalities. Again, it illustrates the importance of the psychophysical paradigm used to study divided attention; that is, detection alone would have left us with the view that each sensory domain has its own processing capacity, in agreement with those (Treisman \& Davies, 1973; Wickens, 1980) who argue against the notion of a single, undifferentiated resource. Instead, the data from identification lend strong support to the idea of a single, higher order limitation on processing at a level that is central to either sensory modality alone. Furthermore, the symmetry between $80 \%, 20 \%$ and $20 \%, 80 \%$ in the AOC suggests that resources withdrawn from one sensory channel were fully applied to the other. Using purely visual tasks, Bonnel et al. (1992) had found the relation between $\left[d^{\prime}\right]^{2}$ and instructions to fit closely to a sample-size model. Similar results here imply that the units of attention were the same for auditory and visual channels. This adds still more credence to the idea of a central bottleneck on attention.

On the basis of comparisons between performance in single tasks and in a variety of dual tasks with the instruction 100\%,0\%, Taylor, Lindsay, and Forbes (1967) suggested that the mere presence of a nonattended source resulted in a "sharing cost" of $15 \%$. This was exactly the cost observed by Bonnel et al. (1992) in their visual identification task. Although the present experiment included no conditions with the instruction $100 \%, 0 \%$, the fact that both single-task and dual-task points fell on the same PRF shows that there was no such cost of sharing 


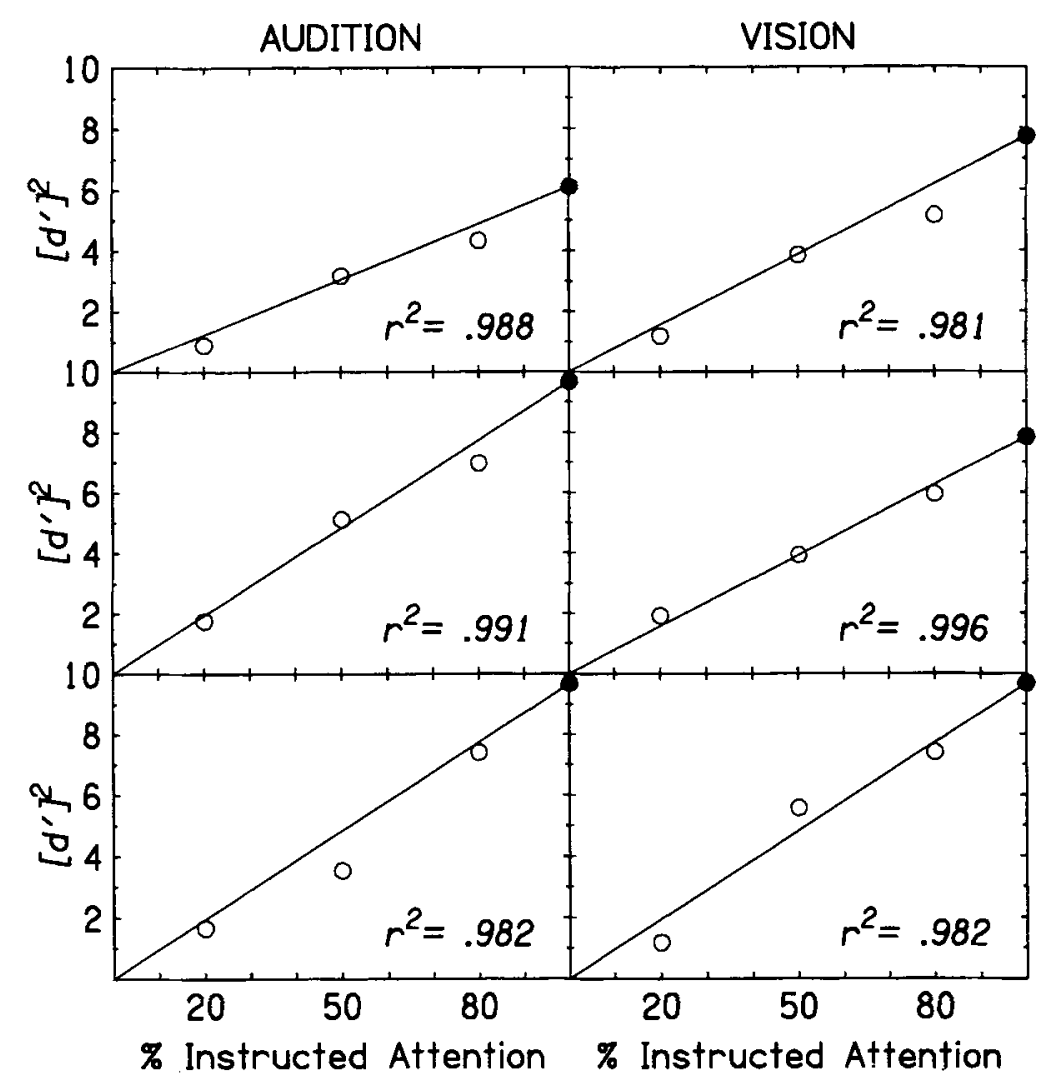

Figure 7. Experiments 1 and 3. PRFs plotting identification performance in units of $\left[d^{\prime}\right]^{2}$ as a function of attentional instructions. Top: Experiment 1. Middle: Experiment 3 with free order of response. Bottom: Experiment 3 with constrained urder of response. Left: auditory channel. Right: visual channel.

in the dual task. This agrees with previous results that have shown that it is easier to monitor two sensory modalities than two channels within a single modality (Proctor \& Proctor, 1979; Treisman \& Davies, 1973).

It is interesting to compare our results with those from others who have examined simultaneous attention to the auditory and visual channels. Although none of the other studies specifically compared detection to identification, this comparison can be made by dividing them into two groups according to the paradigm used. When the tasks were only detection (Brown \& Hopkins, 1967; Eijkman \& Vendrick, 1965; Lindsay, 1970; Shiffrin, 1975; Swets \& Kristofferson, 1970), they found, as we did, that performance was about as good with two channels as with one. Similarly, they also found lower performance with two channels when the task was identification (Long, 1976a, 1976b; Massaro \& Kahn, 1973; Taylor et al., 1967; Tulving \& Lindsay, 1967).

Limitations on divided attention have been the subject of much controversy. The literature has concentrated on stimulus and response factors-more specifically, similarity between channels and responses. The fact that there is a cost of shared attention in identification but not in detection shows that task requirements must also be considered as an important determinant.
Finally, speculations about the site of the limitation in divided attention, when found, have ranged from peripheral to higher order levels of processing. The present data from identification show a tradeoff between resource allocations to two sensory channels. This suggests that for identification, the putative bottleneck operates at a more central level of processing rather than in the auditory and visual peripheries.

\section{REFERENCES}

BonNEl, A. M., \& Miller, J. (1994). Attentional effects on concurrent discriminations: Investigations of a sample-size model. Perception \& Psychophysics, 54, 162-179.

Bonnel, A. M., Possamai, C., \& Schmitt, M. (1987). Early modulations of visual input: A study of attentional strategies. Quarterly Journal of Experimental Psychology, 39A, 757-776.

Bonnel, A. M., Stein, J. F., \& Bertucci, P. (1992). Does attention modulate the perception of luminance changes? Quarterly Journal of Experimental Psychology, 44A, 601-626.

BRown, A. E., \& Hopkins, H. K. (1967). Interactions of the auditory and visual sensory modalities. Journal of the Acoustical Society of America, 41, 1-7.

Dorfman, D. D., \& ALF, E., JR. (1969). Maximum-likelihood estimation of parameters of signal detection theory and determination of confidence intervals: Rating method data. Journal of Mathematical Psychology, 6, 487-496.

DunCaN, J. (1980). The locus of interference in the perception of simultaneous stimuli. Psychological Review, 87, 272-300. 
DuRlach, N. I., \& BRAIDA, L. D. (1969). Intensity perception. I. Preliminary theory of intensity resolution. Journal of the Acoustical Society of America, 46, 372-383.

Egeth, H. (1967). Selective attention. Psychological Review, 67, 41-57.

EIJKMAN, E., \& VENDRICK, A. J. H. (1965). Can a sensory system be specified by its internal noise? Journal of the Acoustical Society of America, 37, 1102-1109.

ElliotT, P. B. (1964/1988). Tables of $d^{\prime}$. In J. Swets (Ed.), Signal detection and recognition by human observers. Contemporary readings (pp. 651-684). Los Altos, CA: Peninsula Publishing.

Gopher, D., \& Navon, D. (1980). How is performance limited: Testing the notion of central capacity. Acta Psychologica, 46, 161-180.

Green, D. M., \& Swets, J. A. (1966). Signal detection and psychophysics. New York: Wiley.

HaYs, W. L. (1969). Statistics. London: Holt, Rinehart \& Winston.

HirsT, W. (1986). Aspects of divided and selective attention. In J. LeDoux \& W. Hirst (Eds.), Mind and brain (pp. 105-141). New York: Cambridge University Press.

HoffMan, J. E., \& NELSON, B. (1981). Spatial selectivity in visual search. Perception \& Psychophysics, 30, 283-290.

Kahneman, D. (1973). Attention and effort. Englewood Cliffs, NJ: Prentice Hall.

KINCHLA, R. A. (1969). An attention operating characteristic in vision (Tech. Rep. No. 29). McMaster University, Hamilton, ON.

Kinchla, R. A. (1980). The measurement of attention. In R. S. Nickerson (Ed.), Attention and performance VIII (pp. 213-218). Hillsdale, NJ: Erlbaum.

KLEIN, S. (1985). Double judgment psychophysics: Problems and solutions. Journal of the Optical Society of America, 2, 1560-1585.

LiNDSAY, P. H. (1970). Multichannel processing in perception. In D. Mostofsky (Ed.), Attention: A behavioral analysis (pp. 149-171). New York: Appleton-Century-Crofts.

LONG, J. (1976a). Division of attention between non-verbal signals: Allor-none or shared processing? Quarterly Journal of Experimental Psychology, 28, 47-69.

LONG, J. (1976b). Division of attention between simultaneous and successive nonverbal signals varying in discriminability. Quarterly Journal of Experimental Psychology, 29, 7-30.

LUCE, D. (1977).Thurstone's discriminal processes 50 years later. Psychometrika, 42, 461-469.

MaCMILlan, N. A. (1971). Detection and recognition of increments and decrements in auditory intensity. Perception \& Psychophysics, 10, 233-238.

Macmillan, N. A. (1973). Detection and recognition of intensity changes in tone and noise: The detection-recognition disparity. Perception \& Psychophysics, 13, 65-75.

Macmillan, N. A., \& Creelman, C. D. (1991). Detection theory: A user's guide. New York: Cambridge University Press.

MaCmillan, N. A., \& Kaplan, H. L. (1985). Detection theory analysis of grouped data: Estimating sensitivity from average hit and falsealarm rates. Psychological Bulletin, 98, 185-199.

MASSARO, D. W., \& KAHN, B. (1973). Effects of central processing on auditory recognition. Journal of Experimental Psychology, 97, 51-58.

Miller, J., \& BonNel, A. M. (1994). Switching or sharing in dual-task line-length discrimination? Perception \& Psychophysics, 56, 431-446.

MülleR, H. J., \& Findlay, J. M. (1987). Sensitivity and criterion effects in the spatial cuing of visual attention. Perception \& Psychophysics, 42, 383-399.

Navon, D. (1985). Attention division or attention sharing? In M. I. Posner \& O. S. M. Marin (Eds.), Attention and performance XI (pp. 133 146). Hillsdale, NJ: Erlbaum.

NAVON, D., \& GopHER, D. (1979). On the economy of the human processing system. Psychological Review, 86, 214-255.

Navion, D., Gopher, D., ChillaG, N., \& SitTz, G. (1984). On separability of and interference between tracking dimensions in dual-task axis. Journal of Motor Behavior, 16, 364-391.

Norman, D. A., \& Bobrow, D. G. (1975). On data-limited and resourcelimited processes. Cognitive Psychology, 7, 44-64.

Proctor, R. W., \& Proctor, J. D. (1979). Secondary tasks modality, expectancy, and the measurement of attentional capacity. Journal of Experimental Psychology: Human Perception \& Performance, 5, 610-624.
REgAN, J. (1982). Short-term memory and dual-task performance. Paper presented at the 26th annual meeting of the Human Factors Society, Seattle.

SCHNEIDER, W., \& Fisk, A. D. (1982). Concurrent automatic and controlled visual search. Can processing occur without resources cost? Journal of Experimental Psychology: Learning, Memory, \& Cognition, 8, 261-278.

SHIFFrIN, R. M. (1975). The locus and role of attention in memory systems. In P. M. A. Rabbitt \& S. Dornic (Eds.), Attention and performance $V$ (pp. 168-191). Hillsdale, NJ: Erlbaum.

SPERLING, G., \& MELCHNER, M. (1978a). The attention operating characteristic: Examples from visual search. Science, 202, 315-318.

SPerling, G., \& Melchner, M. (1978b). Visual search, visual attention, and the attention operating characteristic. In J. Requin (Ed.), Attention and performance VII (pp. 75-686). Hillsdale, NJ: Erlbaum.

Swets, J. A., \& Kristofferson, A. B. (1970). Attention. Annual Review of Psychology, 21, 339-366.

Swets, J. A., \& Pickett, R. M. (1982). Evaluation of diagnostic systems. Methods for signal detection theory analysis. New York: Academic Press.

TAYlOR, M. M., LindSAY, P. H., \& Forbes, S. M. (1967). Quantification of shared capacity processing in auditory and visual discrimination. Acta Psychologica, 27, 223-229.

Treisman, A. M., \& Davies, A. (1973). Dividing attention to ear and eye. In S. Kornblum (Ed.), Attention and performance IV (pp. 101117). New York: Academic Press.

Tulving, E., \& LinDSAY, P. H. (1967). Identification of simultaneously presented simple visual and auditory stimuli. Acta Psychologica, 27, 101-109.

WICKENS, C. D. (1980). The structure of attentional resources. In R. S. Nickerson (Ed.), Attention and performance VIII (pp. 239-257). Hillsdale, NJ: Erlbaum.

\section{NOTES}

1. The total durations of $20-\mathrm{msec}$ for auditory signals included a 5 -msec onset ramp computed as $1 / 2[1+\cos (2 \pi t)]$ for $t=-.5 \rightarrow 0$ and a 5 -msec offset ramp computed as $1 / 2[1+\cos (2 \pi t)]$ for $t=0 \rightarrow$ to .5 .

2. Conceptually, the instruction $100 \%, 0 \%$ should produce a $d_{\mathrm{A}}^{\prime}$ equal to that found in a single-task measurement of $\mathrm{A}$ alone, while $d_{\mathrm{B}}^{\prime}$ should be 0 . However, this is difficult to demonstrate. Subjects say that the instruction is confusing because they cannot imagine responding to a channel while completely ignoring it (Bonnel \& Miller, 1994). For this reason, comparisons here are to true single tasks.

3. The area under the ROC equals the proportion correct by an unbiased observer in a two-interval forced-choice experiment, and is free from assumptions about the underlying distributions (noise and signal + noise).

4. These correlations were also computed for each subject. In identification, $\varphi$ coefficients were quite close to zero. Among the 18 indices ( 6 subjects $\times$ three conditions) 16 were nonsignificant, the remaining two being positive. In detection, none of the coefficients was negative and 10 out of 18 were positive. These correlations can be due to trial-totrial fluctuations that subtly influenced performance on both tasks in the same way (e.g., alertness, practice level) or to the existence of occasional trials on which observers gave wrong answers for reasons extraneous to their perceptual processing (e.g., blinks).

5 . Indeed, only 1 out of the 18 correlations computed for individual subjects was negative $(p<.05)$ and 3 were significantly positive $(p<.05)$.

6 . The concept of a performance resource function was introduced by Norman and Bobrow (1975), although they did not propose a specific metric for the independent variable.

7. When individual contingencies were computed, the significant values were always in the positive direction. In the first identification experiment (free order of response), we found 2 contingency coefficients out of 9 to be positive. In the second part (constrained order of response), 4 out of 12 coefficients were positive. Four coefficients out of 9 were found to be positive in detection (high), and 1 out of 9 in detection (low).

(Manuscript received September 26, 1994; revision accepted for publication February 10, 1997.) 